ISSN 1420-3049

www.mdpi.com/journal/molecules

Article

\title{
Aristolactams and Alkamides of Aristolochia gigantea
}

\author{
Juliana C. Holzbach and Lucia M. X. Lopes*
}

Instituto de Química, Universidade Estadual Paulista, CP 355, 14801-970 Araraquara-SP, Brazil

* Author to whom correspondence should be addressed; E-Mail: lopesxl@iq.unesp.br ;

Tel.: +55-16-3301-6663.

Received: 24 November 2010; in revised form: 16 December 2010 / Accepted: 17 December 2010 / Published: 21 December 2010

\begin{abstract}
A new aristolactam, aristolactam 9- $O-\beta$-D-glucopyranosyl- $(1 \rightarrow 2)-\beta$-Dglucoside, and two alkamides, $\mathrm{N}$-cis- and $\mathrm{N}$-trans-p-coumaroyl-3-O-methyldopamine, were isolated from stems of Aristolochia gigantea, together with the known compounds allantoin, $E$-nerolidol, $\beta$-sitosterol, (+)-kobusin, (+)-eudesmin, trans- $N$-feruloyltyramine, trans- $N$-coumaroyltyramine, trans- $N$-feruloyl-3-O-methyldopamine, aristolactam Ia- $N$ - $\beta$ D-glucoside, aristolactam Ia 8 - $\beta$-D-glucoside, aristolactam IIIa, and magnoflorine. Their structures were determined by spectroscopic analyses.
\end{abstract}

Keywords: Aristolochia gigantea; Aristolochiaceae; aristolactams; alkamides

\section{Introduction}

The Aristolochiaceae family consists of 450 to 600 species, among which more than 200 have been at least partially studied [1]. Most of these studies have focused on a characteristic group of phenanthrenic compounds, which includes the aristolochic acids (AAs) and the aristolactams (ALs), the former of which occur mainly in species of the genus Aristolochia.

In some European countries and until recently in Brazil, Aristolochia herbs have been used in weight-loss regimens. The clinical application of aristolochic acid (AA) has been limited due to its severe nephrotoxic activity. Recent studies have revealed that AA-I can cause direct damage to renal tubular cells, and its carcinigenicity is associated with the formation of promutagenic AA-DNA adducts [2,3]. The cytotoxic potency of AL-I is higher than that of AA-I, and the cytotoxic effects of these molecules are mediated through the induction of apoptosis in a caspase 3-dependent pathway [3]. Consequently many countries have now banned the use of herbs containing AAs and ALs and the US 
Food and Drug Administration has banned the sale of all products that contain AAs and ALs in their formulations [4].

Recently, the aristolactams have received much attention due to an interesting array of biological properties, including anti-inflammatory, antiplatelet, antimycobacterial, and neuro-protective activities [5]. Naturally occurring aristolactams and several synthetic aristolactam derivatives have been shown to have potent antitumor activities against a broad array of human cancer cell lines. Several aristolactams which may possess postcoital antifertility activity have been isolated from Aristolochia indica. In addition, neurological disorders, especially Parkinson's disease, have been treated by administration of the aristolactam taliscanine [6]. Brazilian Aristolochia species, including Aristolochia gigantea, have been used in traditional medicine as abortifacients and in the treatment of wounds and skin diseases [7].

Aristolochia gigantea develops a strong system of subterranean stems and roots (tuberous or rhizomatous roots). $\alpha$-Phellandrene $(60.9 \%)$ and linalool $(16.6 \%)$ are the major constituents of the essential oil obtained from these plant parts [8], whereas germacrene D and $\gamma$-elemene are the most abundant compounds in the leaf oils. trans-Nerolidol and geraniol are the major constituents in the stem and flower oils, respectively [9]. Previous studies on the leaves of this plant have also led to the isolation of allantoin and sitosterol [7], which are also found in significant quantities in other Aristolochiaceae species. In addition, salsolinol, higenamine, and pinitol have been isolated together with several bisbenzylisoquinolinic and 8-benzylberberinic alkaloids from A. gigantea. These latter compounds have an unusual carbon skeleton [7,10,11]. As part of our continuing studies on the Aristolochiaceae family, we report here the isolation and structural elucidation of aristolactams and alkamides, among other compounds, from aerial and ground (rhizomes) stems of A. gigantea.

\section{Results and Discussion}

Compounds 1-15 (Figure 1) were isolated by chromatography and partition procedures from the ethanol extracts of the stems and analyzed by spectrometric methods (IR, UV, MS, 1D- and 2D-NMR). Phytochemical studies on the ethanol extract from rhizomes of $A$. gigantea led to the isolation of 10 known compounds: allantoin (1) [12], E-nerolidol (2) [13,14], $\beta$-sitosterol (3) [15,16], (+)-kobusin (4) [17], (+)-eudesmin (5) [17], trans- $N$-feruloyltyramine (6) [18], aristolactam Ia $N$ - $\beta$-D-glucoside (7) [19], aristolactam Ia 8- $\beta$-D-glucoside (8) [20,21], aristolactam IIIa (9) [22], and magnoflorine (11) [23], together with a new aristolactam (10). In addition, four known compounds, (+)-kobusin (4), trans- $N$-feruloyltyramine (6), trans- $N$-coumaroyltyramine (12) [18], and trans- $N$-feruloyl-3-Omethyldopamine (13) [24], and a mixture of cis and trans new alkamides $(\mathbf{1 4}+\mathbf{1 5})$ were obtained from the aerial stems. The structures of the known compounds were determined by analyses of their physical and spectroscopic data and comparison of these data to those reported in the literature and to those of authentic samples available in our laboratory, which were previously isolated from Aristolochia spp.

A molecular formula of $\mathrm{C}_{29} \mathrm{H}_{31} \mathrm{O}_{15} \mathrm{~N}$ was determined for compound 10 based on its HRMS spectra, which showed quasi-molecular ions at $\mathrm{m} / z 632.1614[\mathrm{M}-\mathrm{H}]^{-}$. The IR spectrum of compound 10 showed characteristic absorption bands of a lactam group at $1,654 \mathrm{~cm}^{-1}$ and hydroxyl groups at 3,442 
and $1,088 \mathrm{~cm}^{-1}$. The DEPT and ${ }^{13} \mathrm{C}$-NMR spectra of $\mathbf{1 0}$ (Table 1) showed signals for 14 aromatic carbons, and acyl ( $\delta \mathrm{c} 167.4)$, methylenedioxy ( $\left.\delta_{\mathrm{c}} 103.0\right)$, and methoxyl $\left(\delta_{\mathrm{C}} 56.0\right)$ groups.

Figure 1. Chemical structures of compounds 1-16.<smiles>NC(=O)NC1NC(=O)NC1=O</smiles><smiles>CCC(CC[C@H](C)C1CCC2C3CC=C4CC(O)CCC4(C)C3CCC21C)C(C)C</smiles>

3<smiles>[R]c1ccc(/C=C/C(=O)NCCc2ccc([R4])c([R])c2)cc1[R]</smiles>

6 OMe $\quad \mathrm{OH} \quad \mathrm{H} \quad \mathrm{OH}$

$\begin{array}{lllllllll}12 & \mathrm{H} & \mathrm{OH} & \mathrm{H} & \mathrm{OH}\end{array}$

$13 \mathrm{OMe} \mathrm{OH}$ OMe $\mathrm{OH}$

$14 \quad \mathrm{H} \quad \mathrm{OH} \quad \mathrm{OMe} \quad \mathrm{OH}$<smiles>COc1cc(CCNC(=O)/C=C\c2ccc(O)cc2)ccc1O</smiles>

15<smiles>C=C[C@](C)(O)CC/C=C(\C)CCC=C(C)C</smiles>

2<smiles>[R]c1ccc([C@@H]2OC[C@H]3[C@H]2CO[C@H]3c2ccc([R3])c([R4])c2)cc1[R]</smiles>

$\begin{array}{ccccc} & \mathrm{R}^{1} & \mathrm{R}^{2} & \mathrm{R}^{3} & \mathrm{R}^{4} \\ 4 & \mathrm{OCH}_{2} \mathrm{O} & \mathrm{OMe} & \mathrm{OMe} \\ 5 & \mathrm{OMe} & \mathrm{OMe} & \mathrm{OMe} & \mathrm{OMe}\end{array}$<smiles></smiles>

$\begin{array}{llll}\mathrm{R}_{1} & \mathrm{R}_{2} & \mathrm{R}_{3} & \mathrm{R}_{4}\end{array}$

7 glc $\quad \mathrm{H} \quad \mathrm{OH} \quad \mathrm{H}$

$8 \mathrm{H} \quad \mathrm{H} \quad$ glc $\mathrm{H}$

$\begin{array}{llllllllll} & \mathrm{H} & \mathrm{H} & \mathrm{H} & \mathrm{OH}\end{array}$

$10 \mathrm{H}$ glc-glc OMe $\mathrm{H}$

$16 \mathrm{H} \quad \mathrm{OMe}$ glc-glc $\mathrm{H}$

The ${ }^{1} \mathrm{H}-\mathrm{NMR}$ spectrum showed signals characteristic of $\mathrm{CON} \underline{\mathrm{H}}$ at $\delta 10.18$ and only four aromatic hydrogens at $\delta 8.26(\mathrm{dd}, J=8.5,1.0), 7.56(\mathrm{dd}, J=8.5,8.0), 7.23(\mathrm{dd}, J=8.0,1.0)$, and $7.65(\mathrm{~s})$. In addition, signals for carbons and hydrogens for a diglycosyl were observed. These data suggested that compound 10 was an aristolactam. ${ }^{1} \mathrm{H}-{ }^{1} \mathrm{H}$ COSY and 1D-TOCSY experiments allowed us to determine that the glycosyl units were $\beta$-glucosyl- $\beta$-glucosyl $(1 \rightarrow 2)$. Furthermore, the negative ESI-MS/MS of the ions at $\mathrm{m} / \mathrm{z} 632.1$ gave rise to ions at $\mathrm{m} / \mathrm{z} 308.1$ and 469.9 that suggested C9-O and O-C1" fragmentations, respectively. The substituent positions on the aristolactam structure were assigned with the help of $g \mathrm{HMBC}$ experiments (Figure 2). These experiments showed correlations between C-9 $(\delta \mathrm{C}$ 
132.6) and $\mathrm{H}-1$ ' $(\delta \mathrm{H} 5.08) ; \mathrm{C}-3(\delta \mathrm{C} 148.2)$ and $\mathrm{C}_{2} \mathrm{O}_{2}(\delta \mathrm{H} 6.46) ; \mathrm{C}-8(\delta \mathrm{C} 157.2)$ and $\mathrm{OC}_{3} \underline{H}_{3}\left(\delta_{\mathrm{H}} 3.94\right)$ and $\mathrm{H}-6\left(\delta_{\mathrm{H}} 7.56\right)$, as well as between $\mathrm{C}-2^{\prime}\left(\delta_{\mathrm{C}} 81.0\right)$ and $\mathrm{H}-1^{\prime \prime}\left(\delta_{\mathrm{H}} 4.65\right)$.

Figure 2. Selected $g \mathrm{HMBC}(\rightarrow)$ correlations and nOe $(\leftrightarrow)$ interactions for aristolactam 10.

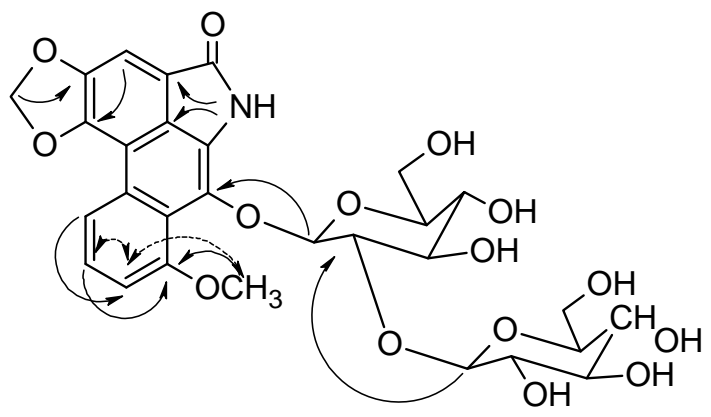

The ${ }^{1} \mathrm{H}$ - and ${ }^{13} \mathrm{C}-\mathrm{NMR}$, IR, and UV spectroscopic data of $\mathbf{1 0}$ were very similar to those reported in the literature for triangularine (16; Figure 1) [25], the main difference being due to interchange of the substituents at C-8 and C-9. The location of the methoxyl group at C-9 in $\mathbf{1 0}$ was corroborated by gNOESY experiments that showed an interaction between $\mathrm{C}_{3} \mathrm{O}$ and $\mathrm{H}-7$. This new aristolactam was named aristolactam 9-O- $\beta$-D-glucopyranosyl- $(1 \rightarrow 2)-\beta$-D-glucoside.

Table 1. NMR data for compound 10. ${ }^{\text {a }}$

\begin{tabular}{cccccc}
\hline Position & $\boldsymbol{\delta}_{\mathbf{H}}$ & $\boldsymbol{\delta}_{\mathrm{C}}$ & Position & $\boldsymbol{\delta}_{\mathbf{H}}$ & $\boldsymbol{\delta}_{\mathrm{C}}$ \\
\hline 1 & & 119.1 & $\mathrm{OCH}_{2} \mathrm{O}$ & $6.46 s$ & 103.0 \\
2 & $7.65 s$ & 105.7 & $\mathrm{OCH}_{3}$ & $3.94 s$ & 56.0 \\
3 & & 148.2 & $1^{\prime}$ & $5.08 d(6.5)$ & 103.0 \\
4 & & 146.9 & $2^{\prime}$ & $3.92 d d(8.5,6.5)$ & 81.0 \\
$4 \mathrm{a}$ & & 109.0 & $3^{\prime}$ & $3.54 t(8.5)$ & 75.8 \\
$4 \mathrm{~b}$ & & 127.9 & $4^{\prime}$ & $3.47 t(8.5)$ & 69.4 \\
5 & $8.26 d d(8.5,1.0)$ & 118.4 & $5^{\prime}$ & $3.18 m$ & 76.9 \\
6 & $7.56 d d(8.0,8.5)$ & 126.1 & $6^{\prime} \alpha, 6^{\prime} \beta$ & $3.8-3.6 \mathrm{~m}$ & 60.5 \\
7 & $7.23 d d(8.0,1.0)$ & 110.8 & $1^{\prime \prime}$ & $4.65 d(7.5)$ & 102.4 \\
8 & & 157.2 & $2^{\prime \prime}$ & $3.06 d d(7.5,8.5)$ & 74.1 \\
$8 \mathrm{a}$ & & 120.0 & $3^{\prime \prime}$ & $3.10 t(8.5)$ & 76.1 \\
9 & & 132.6 & $4^{\prime \prime}$ & $3.17 t(8.5)$ & 69.3 \\
10 & & $\mathrm{~b}$ & $5^{\prime \prime}$ & $2.99 d d d$ & 76.4 \\
& & & & $(8.5,4.7,2.5)$ & \\
$10 \mathrm{a}$ & & 124.4 & $6^{\prime \prime} \alpha, 6^{\prime \prime} \beta$ & $3.35 \mathrm{~m}$ & 60.3 \\
$\mathrm{CO}$ & & 167.4 & $\mathrm{NH}$ & $3.8-3.6 \mathrm{~m}$ & \\
\hline
\end{tabular}

${ }^{\mathrm{a}}$ The ${ }^{1} \mathrm{H}$ - and ${ }^{13} \mathrm{C}-\mathrm{NMR}$ data were assigned with the assistance of $g \mathrm{HMQC}, g \mathrm{HMBC}$, and ${ }^{1} \mathrm{H}-{ }^{1} \mathrm{H}$ COSY experiments $(11.7 \mathrm{~T})$; recorded in DMSO- $d_{6} ; J$ in $\mathrm{Hz} ;{ }^{\mathrm{b}}$ Signal not observed.

The ${ }^{1} \mathrm{H}$ - and ${ }^{13} \mathrm{C}$-NMR spectra of $\mathbf{1 4}+\mathbf{1 5}$ (Table 2) were very similar to those of $\mathbf{1 3}$, except for the absence of a methoxyl group at C-3' in 13, and suggested that it consisted of cis- and trans-alkamides with $p$-disubstituted and trisubstituted aromatic rings. The molecular formula $\left(\mathrm{C}_{18} \mathrm{H}_{19} \mathrm{O}_{4} \mathrm{~N}\right)$ deduced 
from the HRMS spectra was also consistent with the lack of an $\mathrm{OCH}_{3}$ substituent. Based on the integration of the signals corresponding to the olefinic hydrogens $\left[\right.$ cis: $\delta_{\mathrm{H}-2} 5.75(\mathrm{~d}, J=13.0)$ and $\delta_{\mathrm{H}-3}$ $6.48(\mathrm{~d}, J=13.0)$; trans: $\delta_{\mathrm{H}-2} 6.38(\mathrm{~d}, J=15.5)$ and $\left.\delta_{\mathrm{H}-3} 7.28(\mathrm{~d}, J=15.5)\right]$ it was possible to determine that the isolated mixture was in a 1:2 cis/trans proportion. Although cis and trans isomers can isomerize under UV light, both alkamide isomers may be natural compounds [26,27]. To assign with confidence all of the chemical shifts for carbons and hydrogens in the structures, this mixture was exposed to daylight for four hours. The subsequent ${ }^{1} \mathrm{H}-\mathrm{NMR}$ spectrum revealed that the cis/trans proportion had changed to 2:1. The linkage of the methoxyl group to C-3"' was established based on the observation of a correlation between this carbon and the methoxyl hydrogens by $g \mathrm{HMBC}$ experiments, as well as by the spatial interactions of methoxyl hydrogens with H-2"', as observed by 1D-NOESY experiments (Figure 3). Correlations observed by $g \mathrm{HMBC}$ experiments also assisted to determine the carbon skeleton. These alkamides 14 and 15 were named $N$-cis- and $N$-trans- $p$ coumaroyl-3-O-methyldopamine, respectively.

Table 2. NMR data for compounds $\mathbf{1 4}+15 .^{\text {a }}$

\begin{tabular}{ccc}
\hline Position & $\mathbf{1 4} \boldsymbol{\delta}_{\mathbf{H}}$ & $\mathbf{1 5} \boldsymbol{\delta}_{\mathbf{H}}$ \\
\hline 2 & $6.38 d(15.5)$ & $5.75 d(13.0)$ \\
3 & $7.28 d(15.5)$ & $6.48 d(13.0)$ \\
$2^{\prime}, 6^{\prime}$ & $7.36 d(8.5)$ & $7.56 d(8.5)$ \\
$3^{\prime}, 5^{\prime}$ & $6.76 d(8.5)$ & $6.68 d(8.5)$ \\
$2^{\prime \prime}$ & $3.34 m^{\mathrm{b}}$ & $3.34 m^{\mathrm{b}}$ \\
$3^{\prime \prime}$ & $2.62 t(5.5)$ & $2.62 t(5.5)$ \\
$2^{\prime \prime \prime}$ & $6.75 d(2.0)$ & $6.74 d(2.0)$ \\
$5^{\prime \prime \prime}$ & $6.66 d(8.0)$ & $6.66 d(8.0)$ \\
$6^{\prime \prime \prime}$ & $6.59 d d(8.0,2.0)$ & $6.58 d d(8.0,2.0)$ \\
$\mathrm{OCH}_{3}$ & $3.72 s$ & $3.71 s$ \\
$\mathrm{NH}^{\prime \prime}$ & $8.00 t(5.5)$ & $7.98 t(5.5)$ \\
\hline
\end{tabular}

${ }^{\mathrm{a}}$ Recorded in DMSO- $d_{6}, 500 \mathrm{MHz}, J$ in $\mathrm{Hz} ;{ }^{\mathrm{b}}$ Signals assigned with the assistance of ${ }^{1} \mathrm{H}-{ }^{1} \mathrm{H}$ COSY experiments.

Figure 3. Select $g \mathrm{HMBC}(\rightarrow)$ correlations and nOe $(\leftrightarrow)$ interactions for alkamide 14 .

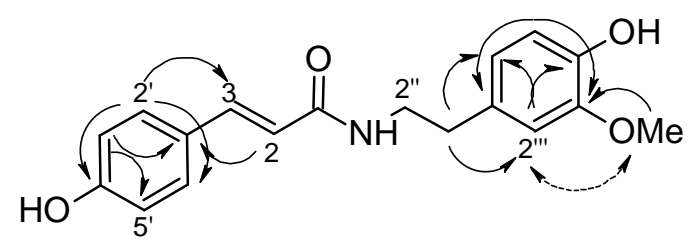

Allantoin (1) is a product of purine metabolism and is widely distributed in biological systems. It has been isolated from marine sponges, animals, and numerous plants, including Aristolochia species. It is used as an anti-inflammatory, antipsoriatic (disputed), and topical vulnerary agent [28]. Allantoin is one of the best-known wound-healing agents, and exerts keratolytic and astringent effects and stimulates new tissue formation [29]. Other well-known compounds that were isolated from $A$. gigantea include $E$-nerolidol, which has been shown to possess larvicidal activity against Aedes 
aegypti [30] and antifungal activity against Microsporum gypseum [31], and magnoflorine, which exhibits insecticidal activity against Spodoptera frugiperda [32], among others activities [33].

\section{Experimental}

\subsection{General}

One-dimensional $\left({ }^{1} \mathrm{H},{ }^{13} \mathrm{C}\right.$, DEPT, and $g$ NOESY) and two-dimensional $\left({ }^{1} \mathrm{H}-{ }^{1} \mathrm{H} g \mathrm{COSY}, g N O E S Y\right.$, $g \mathrm{HMQC}$, and ${ }^{1} \mathrm{H}-{ }^{13} \mathrm{C}$ gHMBC) NMR experiments were performed on a Varian INOVA 500 spectrometer $(11.7 \mathrm{~T})$ at $500 \mathrm{MHz}\left({ }^{1} \mathrm{H}\right)$ and $126 \mathrm{MHz}\left({ }^{13} \mathrm{C}\right)$, using deuterated solvents $\left(\mathrm{CDCl}_{3}\right.$, DMSO$\left.d_{6}\right)(99.98 \% \mathrm{D})$ as an internal standard for ${ }^{13} \mathrm{C}-\mathrm{NMR}$ chemical shifts, and residual solvent as an internal standard for ${ }^{1} \mathrm{H}$ NMR. $\delta$ values are reported relative to TMS. Mass spectra (ESI-MS and ESI-MS/MS) were obtained on a Thermo LCQ, and flow injection into the electrospray source was used for ESIMS. High-resolution mass spectra (HRMS) were obtained on a Bruker Daltonics MicroTOF Ic (ESITOFMS). IR spectra were obtained on a Perkin Elmer FT-IR Spectrum 2000 spectrometer using KBr discs. Optical rotations were measured on a Perkin-Elmer 341-LC polarimeter. Ultraviolet (UV) absorptions were measured on a Perkin-Elmer UV-vis Lambda 14P diode array spectrophotometer. HPLC analyses were performed using a Shimadzu liquid chromatograph (SPD-10 Avp) equipped with UV-Vis and 341-LC polarimeter detectors. RP-18 columns were used (Varian, C18, with a particle size of $5 \mu \mathrm{m}, 250$ by $4.6 \mathrm{~mm}$ for analytical analysis and 250 by $20 \mathrm{~mm}$ for semi-preparative analysis), and chromatograms were acquired at 336 and $254 \mathrm{~nm}$. Melting points were recorded on a Microquímica MQAPF-302 melting point apparatus and are uncorrected.

\subsection{Plant material}

The plant material was collected in Araraquara, SP, Brazil, in February, 2004, and identified as Aristolochia gigantea Mart. (Aristolochiaceae) by Dr. Lindolpho Capellari Júnior (Escola Superior de Agricultura "Luiz de Queiroz" (ESALQ), Piracicaba, SP, Brazil). A voucher specimen (ESA 88281) was deposited at the herbarium of the ESALQ, Piracicaba, SP, Brazil. The material was separated according to the plant parts and dried $\left(c a .45^{\circ} \mathrm{C}\right)$. The stems were further separated into aerial stems and rhizomes.

\subsection{Extraction and isolation of the chemical constituents}

The rhizomes (433.6 g) and aerial stems (379.4 g) were ground and exhaustively extracted successively at room temperature with hexane, acetone, and ethanol. The residues were extracted with ethanol in a Soxhlet apparatus and the extracts were individually concentrated. A portion of the crude ethanol extract of rhizomes $(8.10 \mathrm{~g})$ was washed with $\mathrm{CH}_{3} \mathrm{OH}$. Compound 1 (43.0 mg) was isolated from the insoluble fraction. The methanol-soluble fraction was subjected to CC ( 6.0 by $40.0 \mathrm{~cm}$, silica gel $60 \mathrm{H}, 127.3 \mathrm{~g}$, hexane $/ \mathrm{CH}_{3} \mathrm{OH}$ gradient, $19: 1$ to $\left.100 \% \mathrm{CH}_{3} \mathrm{OH}\right)$ to give 25 fractions (ca. $125 \mathrm{~mL}$ each). Fractions 7, 9, 12, 14, and 15 gave 2 (23.3 mg), 3 (25.0 mg), 4 (409.2 mg), 5 (158.3 mg), and 6 (26.2 mg), respectively. Fraction 23 after HPLC [Varian RP C18 semi-preparative column, eluted with $\mathrm{CH}_{3} \mathrm{OH}-\mathrm{H}_{2} \mathrm{O}+0.5 \% \mathrm{NH}_{4} \mathrm{OH}, 3: 2$, flow $=8 \mathrm{~mL} \mathrm{~min}^{-1} ; \lambda=254 \mathrm{~nm}$ ] gave $1(16.5 \mathrm{mg})$ and 11 $(17.5 \mathrm{mg})$. Fraction $21(1.10 \mathrm{~g})$ was subjected to RP CC (3.0 by $45.0 \mathrm{~cm}$, silica gel C18, $43.5 \mathrm{~g}$, 
$\mathrm{CH}_{3} \mathrm{OH}-\mathrm{H}_{2} \mathrm{O}$ gradient 9:1 to $100 \% \mathrm{CH}_{3} \mathrm{OH}$ ) to give 17 subfractions (ca. $125 \mathrm{~mL}$ each). Subfractions 10 and 11 (98.4 mg) after HPLC [Varian RP C18 column, eluted with $\mathrm{CH}_{3} \mathrm{OH}-\mathrm{H}_{2} \mathrm{O}+0.5 \% \mathrm{NH}_{4} \mathrm{OH} 3: 2$, flow $=8 \mathrm{~mL} \mathrm{~min}^{-1}, \lambda=254 \mathrm{~nm}$ ] gave $7(4.0 \mathrm{mg}), \mathbf{8}(9.6 \mathrm{mg}), \mathbf{9}(1.8 \mathrm{mg})$, and $\mathbf{1 0}(10.1 \mathrm{mg})$. The stem crude ethanol extract $(10.0 \mathrm{~g})$ was fractionated over Sephadex LH-20 (120.0 g, 2.5 by $95.0 \mathrm{~cm}$, $\mathrm{MeOH})$ to give 17 fractions. Fraction $6(166 \mathrm{mg})$ was subjected to two HPLC runs [Varian RP C18 semi-preparative column, eluted with $\mathrm{CH}_{3} \mathrm{OH}-\mathrm{H}_{2} \mathrm{O} 3: 2$, flow $=8 \mathrm{~mL} \mathrm{~min}^{-1}, \lambda=254 \mathrm{~nm}$; followed by Varian RP C18 analytical column, eluted with $\mathrm{CH}_{3} \mathrm{OH}-\mathrm{H}_{2} \mathrm{O} 2: 3$, flow $=0.8 \mathrm{~mL} \mathrm{~min}^{-1}, \lambda=254 \mathrm{~nm}$ ] to give 4 (2.0 mg), 12 (2.0 mg), $13(1.5 \mathrm{mg})$, and $\mathbf{1 4}+\mathbf{1 5}(1.0 \mathrm{mg})]$.

\subsection{Spectral data}

Allantoin (1). Yellow needles. mp 233-234 ${ }^{\circ} \mathrm{C}$ [lit. 232-235 ${ }^{\circ} \mathrm{C}$ ] [12]. IR, ${ }^{1} \mathrm{H}-\mathrm{NMR}$, and ${ }^{13} \mathrm{C}-\mathrm{NMR}$ data were consistent with those previously reported [12].

(-)E-Nerolidol (2). Yellow oil. $[\alpha]_{D}^{25}-17^{\circ}\left(\mathrm{CHCl}_{3}, c\right.$ 0.2) [lit. $-12.5^{\circ}\left(\mathrm{CHCl}_{3}, c\right.$ 0.02)] [13]. ${ }^{1} \mathrm{H}-\mathrm{NMR}$ $\left(\mathrm{CDCl}_{3}\right) \delta 5.15(1 \mathrm{H}, d d, J 17.5,1.5 \mathrm{~Hz}, \mathrm{H}-1 \alpha), 5.00(1 \mathrm{H}, d d, J 10.5,1.5 \mathrm{~Hz}, \mathrm{H}-1 \beta), 5.86(1 \mathrm{H}, d d, J$ $17.5,10.5 \mathrm{~Hz}, \mathrm{H}-2), 1.54-1.51$ (2H, $m, \mathrm{H}-4), 2.00-1.92$ (6H, $m, \mathrm{H}-5, \mathrm{H}-8, \mathrm{H}-9), 5.08-5.01$ (2H, $m, \mathrm{H}-$ 6, H-10), 1.60 (3H, s, H-12), 1.22 (3H, $s, \mathrm{H}-15), 1.54$ (6H, $s, \mathrm{H}-13, \mathrm{H}-14) .{ }^{13} \mathrm{C}-\mathrm{NMR}$ data were consistent with those previously reported [14].

$\beta$-Sitosterol (3). Colorless crystals. $[\alpha]_{D}^{25}-15.2^{\circ}\left(\mathrm{CHCl}_{3}, c\right.$ 0.2) [lit. $\left.-26.1^{\circ}\left(\mathrm{CHCl}_{3}, c 0.1\right)\right][15] .{ }^{13} \mathrm{C}$ NMR data were consistent with those previously reported [16].

(+)-Kobusin (4). Yellow solid. $[\alpha]_{D}^{25}+51.4^{\circ}\left(\mathrm{CHCl}_{3}, c\right.$ 0.21) [lit. $+58.0^{\circ}\left(\mathrm{CHCl}_{3}, c\right.$ 0.03)] [17]. ${ }^{1} \mathrm{H}-$ NMR $\left(\mathrm{CDCl}_{3}\right) \delta 6.83(1 \mathrm{H}, d, J 2.0 \mathrm{~Hz}, \mathrm{H}-2), 6.78\left(1 \mathrm{H}, d, J 2.0 \mathrm{~Hz}, \mathrm{H}-2^{\prime}\right), 6.71(1 \mathrm{H}, d, J 8.0 \mathrm{~Hz}, \mathrm{H}-5)$, $6.77\left(1 \mathrm{H}, d, J 8.0 \mathrm{~Hz}, \mathrm{H}-5^{\prime}\right), 6.81(1 \mathrm{H}, d d, J 2.0,8.0 \mathrm{~Hz}, \mathrm{H}-6), 6.74\left(1 \mathrm{H}, d d, J 2.0,8.0 \mathrm{~Hz}, \mathrm{H}-6^{\prime}\right), 4.67$ $\left(2 \mathrm{H}, d, J 5.5 \mathrm{~Hz}, \mathrm{H}-7 \beta, \mathrm{H}-7^{\prime} \beta\right), 3.02\left(2 \mathrm{H}, m, \mathrm{H}-8 \alpha, \mathrm{H}^{\prime} 8^{\prime} \alpha\right), 4.18\left(2 \mathrm{H}, d d, J 7.0,14.0 \mathrm{~Hz}, \mathrm{H}-9 \beta, \mathrm{H}-9^{\prime} \beta\right)$, $3.82\left(2 \mathrm{H}, m, \mathrm{H}-9 \alpha, \mathrm{H}_{-} 9^{\prime} \alpha\right), 3.83\left(3 \mathrm{H}, s, \mathrm{OCH}_{3}\right), 3.80\left(3 \mathrm{H}, s, \mathrm{OCH}_{3}\right), 5.85\left(2 \mathrm{H}, s, \mathrm{OCH}_{2} \mathrm{O}\right)$.

(+)-Eudesmin (5). Yellow oil. $[\alpha]_{D}^{25}+17.5^{\circ}\left(\mathrm{CHCl}_{3}, c\right.$ 0.12) [lit. $+61^{\circ}\left(\mathrm{CHCl}_{3}, c\right.$ 0.4)] [17]. ${ }^{1} \mathrm{H}-\mathrm{NMR}$ and ${ }^{13} \mathrm{C}-\mathrm{NMR}$ data were consistent with those previously reported [17].

trans- $N$-Feruloyl tyramine (6). Amorphous solid. ${ }^{1} \mathrm{H}-\mathrm{NMR}$ (DMSO- $\left.d_{6}\right) \delta 6.43(1 \mathrm{H}, d, J 15.9, \mathrm{H}-2)$, $7.31(1 \mathrm{H}, d, J 15.9, \mathrm{H}-3), 7.11\left(1 \mathrm{H}, d, J 1.8, \mathrm{H}-2^{\prime}\right), 6.79\left(1 \mathrm{H}, d, J 8.1, \mathrm{H}-5^{\prime}\right), 6.98(1 \mathrm{H}, d d, J 8.1,1.8$, H-6'), 3.32 (2H, $\left.m, \mathrm{H}-2^{\prime \prime}\right), 2.65$ (2H, $\left.t, J 7.2, \mathrm{H}-3^{\prime \prime}\right), 6.68\left(2 \mathrm{H}, d, J 8.5, \mathrm{H}-2^{\prime \prime \prime}, \mathrm{H}-6^{\prime \prime \prime}\right), 7.00$ (2H, $d, J$ 8.5, H-3"', H-5"'), $3.80\left(3 \mathrm{H}, s, \mathrm{OCH}_{3}\right), 7.95(1 \mathrm{H}, t, J 5.7, \mathrm{NH})$.

Aristolactam Ia N- $\beta$-D-glucoside (7). Amorphous solid. $[\alpha]_{D}^{26}-7.9^{\circ}\left(\mathrm{MeOH}, c\right.$ 0.1) [lit. $-9.9^{\circ}(\mathrm{MeOH}$, $c$ 0.07)] [19]. ${ }^{1}$ H-NMR data were consistent with those previously reported [19].

Aristolactam Ia 8- $\beta$-D-glucoside (8). Amorphous solid. $[\alpha]_{D}^{26}-8.2^{\circ}\left(\mathrm{MeOH}, c\right.$ 0.1) [lit. $-10.5{ }^{\circ}$ $(\mathrm{MeOH}, c 0.2)][20] .{ }^{1} \mathrm{H}-\mathrm{NMR}$ data were consistent with those previously reported [20]. 
Aristolactam IIIa (9). Amorphous solid. ${ }^{1} \mathrm{H}-\mathrm{NMR}$ (DMSO-d 6$) \delta 7.62(1 \mathrm{H}, s, \mathrm{H}-2), 7.97(1 \mathrm{H}, d, J 2.5$, $\mathrm{H}-5), 7.10(1 \mathrm{H}, d d, J 2.5,8.5, \mathrm{H}-7), 7.79(1 \mathrm{H}, d, J 8.5, \mathrm{H}-8), 7.05(1 \mathrm{H}, s, \mathrm{H}-9), 6.48\left(2 \mathrm{H}, s, \mathrm{OCH}_{2} \mathrm{O}\right)$, $10.65(1 \mathrm{H}, s, \mathrm{NH})$.

Aristolactam 9-O- $\beta$-D-glucopyranosyl- $(1 \rightarrow 2)-\beta$-D-glucoside (10). Amorphous solid. $[\alpha]_{D}^{26}-3.9{ }^{\circ}(c$ 0.5, MeOH). ${ }^{1} \mathrm{H}-\mathrm{NMR}\left(\mathrm{CDCl}_{3}\right)$ and ${ }^{13} \mathrm{C}-\mathrm{NMR}\left(\mathrm{CDCl}_{3}\right)$ spectra see Table 1; ESI-HR-TOF-MS (probe), $4000 \mathrm{~V}, \mathrm{~m} / z$ (rel. int.): $632.1614[\mathrm{M}-\mathrm{H}]^{-}$(100) (calculated for $\mathrm{C}_{29} \mathrm{H}_{31} \mathrm{O}_{15} \mathrm{~N}-\mathrm{H}=632.1615$ ); ESIMS/MS (probe) 4,500 V from ions at $m / z 632.1$ (100), $m / z$ (rel. int.): 308.1 [M - glc-glc $]^{-}$(92).

Magnoflorine (11). Amorphous solid. $[\alpha]_{D}^{25}+164.4^{\circ}\left(\mathrm{MeOH}, c\right.$ 0.03) [lit. $+150.0^{\circ}(\mathrm{MeOH}, c$ 0.1)] [23]. ${ }^{1} \mathrm{H}-\mathrm{NMR}$ (DMSO- $\left.d_{6}\right) \delta 6.49$ (1H, $\left.s, \mathrm{H}-3\right), 2.7-2.8(2 \mathrm{H}, m, \mathrm{H}-4 \alpha, \mathrm{H}-4 \beta), 3.7-3.6$ (2H, $m, \mathrm{H}-5 \alpha$, $\mathrm{H}-5 \beta), 4.34$ (1H, $b r d, J 13.0, \mathrm{H}-6 \mathrm{a}), 2.59(1 \mathrm{H}, t, J 13.0, \mathrm{H}-7 \alpha), 3.10(1 \mathrm{H}, b r d, J 13.0, \mathrm{H}-7 \beta), 6.35$ $(1 \mathrm{H}, b r d, J 8.0, \mathrm{H}-8), 6.59(1 \mathrm{H}, d, J 8.0, \mathrm{H}-9), 3.65\left(3 \mathrm{H}, s, \mathrm{OCH}_{3}-10\right), 3.68\left(3 \mathrm{H}, s, \mathrm{OCH}_{3}-2\right), 2.88(3 \mathrm{H}$, $\left.s, \mathrm{~N}-\mathrm{CH}_{3}\right), 3.29\left(3 \mathrm{H}, s, \mathrm{~N}-\mathrm{CH}_{3}\right) .{ }^{13} \mathrm{C}-\mathrm{NMR}\left(\mathrm{DMSO}-d_{6}\right) \delta 152.1(\mathrm{C}-1), 151.2(\mathrm{C}-2), 108.8(\mathrm{C}-3), 111.6$ (C-3a), 23.2 (C-4), 60.5 (C-5), 69.2 (C-6a), 30.4 (C-7), 125.1 (C-7a), 112.4 (C-8), 109.9 (C-9), 150.3 (C-10), 152.5 (C-11), 122.6 (C-11a), 123.1 (C-1a), 120.0 (C-1b), 55.2, $55.7\left(\mathrm{OCH}_{3}\right), 42.5,52.6$ $\left(\mathrm{N}-\mathrm{CH}_{3}\right)$.

trans-N-Coumaroyltyramine (12). ${ }^{1} \mathrm{H}-\mathrm{NMR}\left(\mathrm{DMSO}_{6}\right) \delta 6.38(1 \mathrm{H}, d, J 15.9, \mathrm{H}-2), 7.30(1 \mathrm{H}, d, J$ 15.9, H-3), 7.37 (2H, $d, J$ 8.7, H-2', H-6'), 6.78 (2H, $d, J$ 8.7, H-3', H-5'), 3.31 (2H, $\left.m, \mathrm{H}-2^{\prime \prime}\right), 2.64$ (2H, $t, J$ 7.2, H-3"), 7.00 (2H, d, J 8.4, H-2'"', H-6"' ), 6.67 (2H, $d, J$ 8.4, H-3"', H-5"'), 7.94 (1H, $m, \mathrm{NH})$.

trans-N-Feruloyl-3-O-methyldopamine (13). ${ }^{1} \mathrm{H}-\mathrm{NMR}$ (DMSO- $\left.d_{6}\right) \delta 6.43(1 \mathrm{H}, d, J 15.3, \mathrm{H}-2), 7.30$ $(1 \mathrm{H}, d, J 15.3, \mathrm{H}-3), 7.10\left(1 \mathrm{H}, d, J 2.1, \mathrm{H}-2^{\prime}\right), 6.78\left(1 \mathrm{H}, d, J 7.8, \mathrm{H}-5^{\prime}\right), 6.97\left(1 \mathrm{H}, d d, J 7.8,2.1, \mathrm{H}-6^{\prime}\right)$, $3.32\left(2 \mathrm{H}, m, \mathrm{H}-2^{\prime \prime}\right), 2.65$ (2H, $t, J$ 6.9, H-3"'), 6.77 (1H, $d, J$ 2.1, H-2'"'), 6.68 (1H, $\left.d, J 7.8, \mathrm{H}-5^{\prime \prime \prime}\right), 6.60$ $\left(1 \mathrm{H}, d d, J 7.8,2.1, \mathrm{H}-6^{\prime \prime \prime}\right), 3.79\left(3 \mathrm{H}, s, \mathrm{OCH}_{3}\right), 3.74\left(3 \mathrm{H}, s, \mathrm{OCH}_{3}\right), 7.94(1 \mathrm{H}, m, \mathrm{NH})$.

$\mathrm{N}$-cis- and $\mathrm{N}$-trans-p-Coumaroyl-3-O-methyldopamine $(\mathbf{1 4}+\mathbf{1 5})$. Colorless oil. ${ }^{1} \mathrm{H}-\mathrm{NMR}\left(\mathrm{CDCl}_{3}\right)$ spectra see Table 1. ${ }^{13} \mathrm{C}-\mathrm{NMR}\left(\mathrm{DMSO}-d_{6}\right) \delta 137.8(\mathrm{C}-3), 127.0\left(\mathrm{C}-2^{\prime}, 6^{\prime}\right), 116.0\left(\mathrm{C}-3^{\prime}, 5^{\prime}\right), 113.2(\mathrm{C}-$

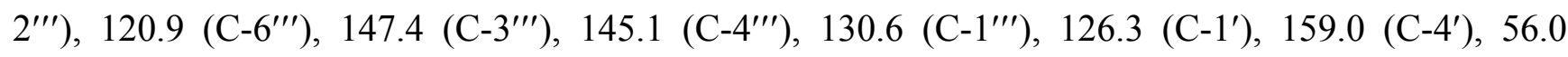
$\left(\mathrm{OCH}_{3}\right)$. ESI-HR-TOF-MS (probe) 4,000V, $\mathrm{m} / z$ (rel. int.): $312.1233[\mathrm{M}-\mathrm{H}]^{-}$(100) (calculated for $\left.\mathrm{C}_{18} \mathrm{H}_{19} \mathrm{O}_{4} \mathrm{~N}-\mathrm{H}=312.1236\right)$.

\section{Conclusions}

Extracts from different parts of $A$. gigantea showed a diverse chemical composition. As previously observed, the characteristic chemical constituents of the leaves of this species are bisbenzylisoquinolinic and 8-benzylberberinic alkaloids, whereas stems contain lignans at high concentrations, alkamides, and aristolactams. Among the compounds that were isolated from stems, two alkamides and an aristolactam are described here for the first time. 


\section{Acknowledgements}

The authors thank Condorcet Aranha and Lindolpho Capellari Jr. for plant identification. Thank the Fundação de Amparo à Pesquisa do Estado de São Paulo (FAPESP) and Conselho Nacional de Desenvolvimento Científico e Tecnológico (CNPq/MCT/MS/PRONEX, Brazil) for financial support, and the Coordenação de Aperfeiçoamento de Nível Superior (CAPES) for providing a fellowship to J.C.H.

\section{References and Notes}

1. Lopes, L.M.X.; Nascimento, I.R.; Silva, T. Phytochemistry of the Aristolochiaceae Family. In Research Advances in Phytochemistry; Mohan, R.M.M., Ed.; Global Research Network: Kerala, India, 2001; Volume 2, pp. 19-108.

2. Li, Y.; Liu, Z.; Guo, X.; Shu, J.; Chen, Z.; Li, L. Aristolochic Acid I-induced DNA Damage and Cell Cycle Arrest in Renal Tubular Epithelial Cells in Vitro. Arch. Toxicol. 2006, 80, 524-532.

3. Li, J.; Zhang, L.; Jiang, Z.; Shu, B.; Li, F.; Bao, Q.; Zhang, L. Toxicities of Aristolochic Acid I and Aristololactam I in Cultured Renal Epithelial Cells. Toxicol. In Vitro 2010, 24, 1092-1097.

4. Zhang, J.; Xiao, Y.; Feng, J.; Wu, S.L.; Xue, X.; Zhang, X.; Liang, X. Selectively Preparative Purification of Aristolochic Acids and Aristololactams from Aristolochia Plants. J. Pharm. Biomed. Anal. 2010, 52, 446-451.

5. Choi, Y.L.; Kim, J. K.; Choi, S.-U.; Min, Y.-K.; Bae, M.-A.; Kim, B.T.; Heo, J.-N. Synthesis of Aristolactam Analogues and Evaluation of Their Antitumor Activity. Bioorg. Med. Chem. Lett. 2009, 19, 3036-3040.

6. Kumar, V.; Prasad, A.K.; Parmar, V.S. Naturally Occurring Aristolactams, Aristolochic Acids and Dioxoaporphines and Their Biological Activities. Nat. Prod. Rep. 2003, 20, 565-583.

7. Lopes, L.M.X.; Humpfer, E. 8-Benzylberbine and N-Oxide Alkaloids from Aristolochia gigantea. Phytochemistry 1997, 45, 431-435.

8. Francisco, C.S.; Messiano, G.B.; Lopes, L.M.X.; Tininis, A.G.; de Oliveira, J.E.; Capellari, L., Jr. Classification of Aristolochia Species Based on GC-MS and Chemometric Analyses of Essential Oils. Phytochemistry 2008, 69, 168-175.

9. Leitão, G.G.; Lopes, D.; Menezes, F.D.S.; Kaplan, M.A.C.; Craveiro, A.A.; Alencar, J.W. Essential Oils from Brazilian Aristolochia. J. Essent. Oil Res. 1991, 3, 403-408.

10. Cortes, D.; Dadoun, H.; Paiva, R.L.R.; de Oliveira, A.B. Nouveaux Alcaloïdes Bisbenzylisoquinoleiques Isoles des Feuilles de Aristolochia gigantea. J. Nat. Prod. 1987, 50, 910-914.

11. Lopes, L.M.X. 8-Benzylberbine Alkaloids from Aristolochia gigantea. Phytochemistry 1992, 31, 4005-4009.

12. Sang, S.; Lao, A.; Wang, H.; Chen, Z.; Uzawa, J.; Fujimoto, Y. A Phenylpropanoid Glycoside from Vaccaria segetalis. Phytochemistry 1998, 48, 569-571.

13. Cane, D.E.; Ha, H.-J.; Mcllwaine, D.B.; Pascoe, K.O. The Synthesis of (3R)-Nerolidol. Tetrahedron Lett. 1990, 31, 7553-7554.

14. Suarez, L.E.C.; Menichini, F.; Monache, F.D. Tetranortriterpenoids and Dihydrocinnamic Acid Derivatives from Hortia colombiana. J. Braz. Chem. Soc. 2002, 13, 339-344. 
15. Nakabayashi, R.; Kusano, M.; Kobayashi, M.; Tohge, T.; Yonekura-Sakakibara, K.; Kogure, N.; Yamazaki, M.; Kitajima, M.; Saito, K.; Takayama, H. Metabolomics-oriented Isolation and Structure Elucidation of 37 Compounds Including Two Anthocyanins from Arabidopsis thaliana. Phytochemistry 2009, 70, 1017-1029.

16. Nes, W.D.; Norton, R.A.; Benson, M. Carbon-13 NMR Studies on Sitosterol Biosynthesized from $\left[{ }^{13} \mathrm{C}\right]$ Mevalonates. Phytochemistry 1992, 31, 805-811.

17. Latip, J.; Hartley, T.G.; Waterman. P.G. Lignans and Coumarins Metabolites from Melico pehayesii. Phytochemistry 1999, 51, 107-110.

18. Chang, Y.-C.; Chen, C.-Y.; Chang, F.-R.; Wub, Y.-C. Alkaloids from Lindera glauca. J. Chin. Chem. Soc. 2001, 48, 811-815.

19. Leu, Y.-L.; Chan, Y.-Y.; Hsu, M.-Y.; Chen, I.-S.; Wu, T.-S. The Constituents of the Stem and Roots of Aristolochia foveolata. J. Chin. Chem. Soc. 1998, 45, 539-541.

20. Zhang, Y.T.; Jiang, J.Q. Alkaloids from Aristolochia manshuriensis (Aristolochiaceae). Helv. Chim. Acta 2006, 89, 2665-2670.

21. Hegde, V.R.; Borges, S.; Patel, M.; Das, P.R.; Wu, B.; Gullo, V.P.; Chan, T.-M. New Potential Antitumor Compounds from the Plant Aristolochia manshuriensis as Inhibitors of the CDK2 Enzyme. Bioorg. Med. Chem. Lett. 2010, 20, 1344-1346.

22. Priestap, H.A. Seven Aristolactams from Aristolochia argentina. Phytochemistry 1985, 24, 849-852.

23. Chen, J.-H.; Du, Z.-Z.; Shen, Y.-M.; Yang, Y.-P. Aporphine Alkaloids from Clematis parviloba and their Antifungal Activity. Arch. Pharm. Res. 2009, 32, 3-5.

24. Cutillo, F.; D’Abrosca, B.; DellaGreca, M.; Di Marino, C.; Golino, A.; Previtera, L.; Zarrelli, A. Cinnamic Acid Amides from Chenopodium album: Effects on Seeds Germination and Plant Growth. Phytochemistry 2003, 64, 1381-1387.

25. Lin, W.-H.; Fu, H.-Z.; Hano, Y.; Nomura, T. Alkaloids from the Roots of Aristolochia Triangularis (I). J. Chin. Pharm. Sci. 1997, 6, 8-13.

26. Pedersen, H.A.; Steffensen, S.K.; Christophersen, C. Cinnamoylphenethylamine ${ }^{1}$ H-NMR Chemical Shifts: A Concise Reference for Ubiquitous Compounds. Nat. Prod. Commun. 2010, 5 , 1259-1262.

27. Navickiene, H.M.D.; Lopes, L.M.X. Alkamides and Phenethyl Derivatives from Aristolochia gehrtii. J. Braz. Chem. Soc. 2001, 12, 467-472.

28. Ferreira, D.T.; Alvares, P.S.M.; Houghton, P.J.; Braz-Filho, R. Constituintes Químicos das Raízes de Pyrostegia Venusta e Considerações Sobre a sua Importância Medicinal. Quim. Nova 2000, 23, 42-46.

29. Lee, M.-Y.; Lee, N.-H.; Jung, D.; Lee, J.-A.; Seo, C.-S.; Lee, H.; Kim, J.-H.; Shin, H.-K. Protective Effects of Allantoin Against Ovalbumin (OVA)-Induced Lung Inflammation in a Murine Model of Asthma. Int. Immunopharmacol. 2010, 10, 474-480.

30. Simas, N.K.; Lima, E.C.; Conceição, S.R.; Kuster, R.M.; Oliveira, A.M. Produtos Naturais para o Controle da Transmissão da Dengue: Atividade Larvicida de Myroxylon balsamum (óleo vermelho) e de Terpenóides e Fenilpropanóides. Quim. Nova 2004, 27, 46-49.

31. Shen, Y.; Li, C.G.; Zhou, S.F.; Pang, E.C.K.; Story, D.F.; Xue, C.C.L. Chemistry and Bioactivity of Flos Magnoliae, a Chinese Herb for Rhinitis and Sinusitis. Curr. Med. Chem. 2008, 15, 1616-1627. 
32. Tringali, C.; Spatafora, C.; Calì, V.; Simmonds, M.S.J. Antifeedant Constituents from Fagara macrophylla. Fitoterapia 2001, 72, 538-543.

33. Yokozawa, T.; Satoh, A.; Cho, E.J.; Kashiwada, Y.; Ikeshiro, Y. Protective Role of Coptidis Rhizoma Alkaloids Against Peroxynitrite-induced Damage to Renal Tubular Epithelial Cells. J. Pharm. Pharmacol. 2005, 57, 367-374.

Sample Availability: Samples of the compounds are available from the authors.

(C) 2010 by the authors; licensee MDPI, Basel, Switzerland. This article is an open access article distributed under the terms and conditions of the Creative Commons Attribution license (http://creativecommons.org/licenses/by/3.0/). 